\title{
Whiteboards: important part of the toolbox for improving patient understanding during hospitalisation
}

\author{
Sara Dunbar, ${ }^{1}$ Kathlyn E Fletcher $^{1,2}$
}

'Internal Medicine, Medical College of Wisconsin, Milwaukee, Wisconsin, USA ${ }^{2}$ Internal Medicine, Clement J. Zablocki VAMC, Milwaukee, Wisconsin, USA

\section{Correspondence to}

Dr Kathlyn E Fletcher, Internal Medicine, Clement J. Zablocki VAMC, Milwaukee, WI 53295 USA; kfletche@mcw.edu

Accepted 25 February 2020 Published Online First 5 March 2020

\section{Linked}

- http://dx.doi.org/10.1136/ bmjqs-2019-010208

\section{Check for updates}

(C) Author(s) (or their employer(s)) 2020. No commercial re-use. See rights and permissions. Published by BMJ.

To cite: Dunbar $S$, Fletcher KE. BMJ Qual Saf 2020;29:785-786.
'What are effective strategies to identifying and overcoming barriers to comprehension of information delivered to patients during hospitalisation and at discharge?' This is one of 11 priority questions identified through the Improving Hospital Outcomes through Patient Engagement Study, a 2018 Patient-Centered Outcomes Research Institute (PCORI)funded project to define a patient-centred research agenda for hospital medicine. ${ }^{1}$ In this issue of BMJ Quality \& Safety, Goyal et al explored the use of whiteboards in patient rooms as one such strategy.

The article, entitled 'Do Beside Whiteboards Enhance Communication in Hospitals? An Exploratory Multi-Method Study of Patient and Nurse Perspectives', examined the perspectives of both patients and nurses on whiteboard use. ${ }^{2}$ Over a year, the team surveyed almost 500 adults admitted at the University of Michigan Hospital in order to elicit their views on usability and content of whiteboard information. The authors also included nurses' perspectives gathered through a focus group. Both patients and nurses felt that whiteboards were an important communication tool. However, they identified opportunities for improvement too.

One way to better use whiteboards for communication and information sharing may be to actively encourage patient involvement in the use of the whiteboards. Most nurses in the Goyal et al study felt responsible for maintaining the whiteboard, and only $60 \%$ of patients reported being aware they could use the board to pose questions to the care team. ${ }^{2}$ Perhaps care teams could physically give patients a whiteboard marker at admission as a way to empower them to use the whiteboard. If the patient is unable to do so, the nurse or medical assistant could meet with the patient or their caregiver each morning and write questions on their behalf. For this to be effective, physicians would need to ensure daily review of the patient questions on the whiteboard and discuss as well as write responses for the patient and family. This raises the concern of adequate space on the whiteboards, legibility of writing, and accidental removal of patient questions and/or physician responses.

In the Goyal et al study, $80 \%$ of patients felt that the whiteboard was useful for alerting them to upcoming tests. ${ }^{2}$ An alternative method for sharing such information with patients is by providing a paper outline of the daily inpatient itinerary. The daily plan was created at the veterans administration to provide a detailed summary of what is planned on each hospital day. ${ }^{3}$ The daily plan is a printed document that may include medications, diagnostic testing, appointments and allergies. Patients, their caregivers and nursing staff review the plan together each morning, enabling patients to be actively involved in their own care and to identify any incongruities with their care plan. Most patients (75\%) agreed or strongly agreed that having the daily plan helped them feel more comfortable, increased their understanding and made it easier for them to ask questions. Ideally, the daily plan would be reviewed with patients prior to physicians rounding, so patients and their families could clarify the plan and ask questions when the provider is present. Perhaps they could even use the whiteboards to pose such questions to the physician teams to answer during rounds. One potential drawback to the the daily plan is that when changes and delays in scheduled appointments (inevitably) occur, they could lead to more frustration for patients and their families.

In order to continue to improve communication and patient engagement 
among patients and their providers during hospitalisation, inpatient portals, such as Epic's MyChart Bedside, have also been created for patients to access their medical records via a computer, tablet or phone. Online portals are common in the outpatient setting, and they are becoming increasingly more prevalent in the inpatient setting. Several components can be included in the inpatient portals, including dining options, scheduling information, medications, recent laboratory results, photos of the care team and secure messaging to providers.

McAlearney et al studied the use of MyChart Bedside and demonstrated that patients mainly used the inpatient portal to order meals, view health information and see pictures of their care teams. ${ }^{4}$ Within the online portal, patients would have the ability to see their vitals and laboratory results in real time. Patients can also review their medications for accuracy and identify discrepancies between outpatient and inpatient medications. Patient access to this information creates the necessity for communication channels back to the team for questions and the resolution of discrepancies. MyChart Bedside gives patients and families the option to message providers any questions or clarifications on medications, results or treatment plans. Such questions could also be posed during rounds or on whiteboards. McAlearney et al found portals to be helpful in four ways: promoting independence, reducing anxiety, informing families and empowering patients.

Several concerns with inpatient portals for sharing health information and communication have been raised. First, patients should receive the right amount of information that is meaningful to them, which is likely to differ between patients. If too much information is shared, this could be overwhelming and provoke more uncertainty and anxiety for patients and their families. For example, if laboratory results are released when completed, patients may be concerned about any deviations from the normal range and may not understand the meaning of their results. Second, some patients may find the technology aspect of a tablet or phone challenging despite education on the use of the portal. Many factors related to both patients (eg, age and race) and portals (eg, usability and provider encouragement) affect the adoption of such technology by individual patients. ${ }^{5}$ Third, the messaging feature is meant to improve communication and access to providers; however, in the McAlearney et al survey, most patients indicated they did not use the secure messaging feature. In addition, providers will not be available to answer questions at all times, which could delay viewing questions and providing answers. While these delays may not be a problem in the outpatient setting, the 24/7 nature of inpatient care may make delayed responses frustrating for patients. While the main goal of inpatient portals is to improve patient communication and engagement with healthcare, secure messaging may not always be the answer. Therefore, it is a good idea to have other options for closing the loop on questions that arise from information provided via portals.

Patients have different ways of gathering, processing and retaining health information. ${ }^{6}$ A single method of communication will not work for all patients. Strategies to optimise communication and encourage active patient involvement should take into consideration patients' preferences. For many patients, a combination of communication modalities may be appropriate. This current study has laid the groundwork for the next generation of whiteboards. Using them to identify patient informational needs, answer patient questions and keep patients apprised of hospital plans is a solid start. Combining whiteboards with other printed plans or online technology may be an even stronger way to tailor care to the needs of individual patients.

Funding The authors have not declared a specific grant for this research from any funding agency in the public, commercial or not-for-profit sectors.

Competing interests None declared.

Patient consent for publication Not required.

Provenance and peer review Commissioned; internally peer reviewed.

\section{REFERENCES}

1 Society of Hospital Medicine. Improving hospital outcomes through patient engagement: the i-HOPE study. Philadelphia, PA, 2020. https://www.hospitalmedicine.org/clinical-topics/ihope-study/

2 Goyal A, Glanzman H, Quinn M, et al. Do bedside whiteboards enhance communication in hospitals? an exploratory multimethod study of patient and nurse perspectives. BMJ Qual Saf 2020;29:794-801.

3 King BJ, Mills PD, Fore A, et al. The daily Plan ${ }^{\circledR}$ : including patients for safety's sake. Nurs Manage 2012;43:15-18.

4 McAlearney AS, Fareed N, Gaughan A, et al. Empowering patients during hospitalization: perspectives on inpatient portal use. Appl Clin Inform 2019;10:103-12.

5 Irizarry T, DeVito Dabbs A, Curran CR. Patient portals and patient engagement: a state of the science review. J Med Internet Res 2015;17:e148.

6 Inott T, Kennedy BB. Assessing learning styles: practical tips for patient education. Nurs Clin North Am 2011;46:313-20. 